\title{
Applications of Gauge-fixed Correlation Functions of Quarks and Gluons *
}

\author{
Claude Bernard, ${ }^{a, c}$ Amarjit Soni, ${ }^{a, b}$ Ken Yee ${ }^{b}$
}

\section{DISCLAIMER}

\begin{abstract}
This report was prepared as an account of work sponsored by an agency of the United States Government. Neither the United States Government nor any agency thereof, nor any of their employees, makes any warranty, express or implied, or assumes any legal liability or responsibility for the accuracy, completeness, or usefulness of any information, apparatus, product, or process disclosed, or represents that its use would not infringe privately owned rights. Reference herein to any specific commercial product, process, or service by trade name, trademark, manufacturer, or otherwise does not necessarily constitute or imply its endorsement, recommendation, or favoring by the United States Government or any agency theseof. The views and opinions of authors expressed herein do not necessarily state or reflect those of the United States Government or any agency thereof.
\end{abstract}

* Presented by K. Yee at Lattice '90, International Conference on Lattice Field Theory, Tallahassee, Florida, October 8-12, 1990

a Visiting Appointment: Institute of Theoretical Physics, Santa Barbara, CA 93106

b Physics Department, Brookhaven National Laboratory, Upton, NY 11973

c Address after January 1, 1991: Department of Physics, Washington University, St. Louis, MO 63130

This manuscript has been authored under contract number DE-AC02-76Cll00016 with the U.S. Department of Energy. Accordingly, the U.S. Government retains a non-exclusive, royalty-free license to publish or reproduce the published form of this contribution, or allow others to do so, for U.S. Government purposes. 


\title{
APPLICATIONS OF GAUGE-FIXED
}

\section{CORRELATION FUNCTIONS OF QUARKS AND GLUONS *}

\author{
Claude BERNARD ${ }^{a}$ Amarjit SONI ${ }^{a, b}$, Ken YEE \\ anstitute of Theoretical Physics, Santa Barbara, CA 93106, USA \\ 'Physics Department, Brookhaven National Laboratory, Upton, NY 11973, USA ‡
}

Two uses of fixed gauge correlation functions involving quarks and gluons- as probes of effective quark and gluon parameters and their gauge dependence and as constraints for matching coefficients-are examined. Effective quark and gluon masses are found to decrease with increasing gauge parameter $\lambda$. A fixed gauge technique is used to determine $\bar{s} d$ matching coefficients. Despite gauge variance of effective quark masses, results of this matching - modulo statistics-are gauge invariant. Accordingly this technique is applied to the Delta $I=1 / 2$ Rule. We are however unable to obtain physically useful results at present in this case because of large fluctuations.

\section{INTRODUCTION}

Computation of gauge variant correlation functions in lattice QCD has been the subject of several recent papers. $1,2,3$ The gluon propagator was studied in Ref. 1 and the quark propagator was studied in Ref. 2 on quenched Wilson lattices. Beyond demonstrating that quark and gluon propagators have sensible behavior containing apparent mass gaps even if quarks and gluons are not experimentally observable states, these studies display what may be fundamental features of nonperturbative QCD. Indeed, the effective quark mass is found to decrease linearly with decreasing $m_{\pi}^{2}$ to a critical value which is nonzero. ${ }^{2}$

Here we examine two more uses for quark and gluon correlation functions:

- I. as probes for effective quark/gluon masses and vertices and their gauge dependence;

- II. as constraints for matching between lattice and continuum operators.

For these applications, the following fixed-gauge correlation functions are being computed on the lattice:

$$
\text { - }<0|\psi(x) \bar{\psi}(0)| 0>
$$

$$
\begin{array}{ll}
\text { - } & <0\left|A^{\mu}(x) A^{\nu}(0)\right| 0> \\
\text { - } & <0\left|\prod_{0 \leq t^{\prime} \leq t} U_{i}\left(\vec{x}, t^{\prime}\right)\right| 0> \\
\text { - } & <0|\psi(y) \bar{\psi}(0) \Gamma \psi(0) \bar{\psi}(x)| 0> \\
\text { - } & <0|s(y)(\bar{s}(0) d(0)) \bar{d}(x)| 0> \\
\text { - } & <0\left|s(y) O_{ \pm}\left[\Gamma_{1} \Gamma_{2}\right] \bar{d}(x)\right| 0>\text {. }
\end{array}
$$

Effective quark and gluon masses are extracted by projecting (1) and (2) down to their zero momentum, physical polarizations and averaging the log-ratio of successive propagator timeslices over the "plateau." 2 Global symmetries suppress all components of (3) except

$$
\frac{1}{3} \operatorname{Re} \operatorname{Tr}<0\left|\prod_{0 \leq t^{\prime} \leq t} U_{\hat{t}}\left(\vec{x}, t^{\prime}\right)\right| 0>\equiv Z_{h} e^{-V_{h} t}
$$

where $V_{h}$ may be interpreted as $\left(M_{q}-m_{\text {curr }}\right)$ of a static heavy quark. Function (4) gives for arbitrary Dirac matrix $\Gamma$ the quark's effective electromagnetic radius, magnetic moment, and other couplings. ${ }^{4}$ Functions (5) and (6) are needed for application II, described below.

We present here results from two lattices: sixteen $\beta=5.716^{3} \times 24$ gauge configurations $\left(16^{3} \times 39\right.$ quark propagators) and eight $\beta=6.0$ $24^{3} \times 40$ gauge configurations $\left(24^{3} \times 39\right.$ quark

"Presented by K. Yee at LAT '90, Tallahassee, Florida, October, 1990.

†Address after Jan 1, 1991: Dept. of Physics, Washington University. St. Louis, MO 63130

‡BNL is operated by Associated Universities, Inc. under contract with U.S. Department of Energy 


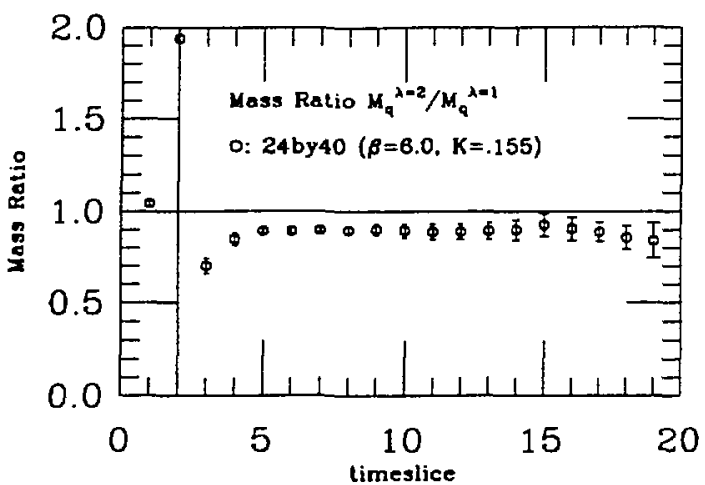

Figure 1: Ratios of effective masses of $\lambda=2$ to $\lambda=1$. Despite the fact that the individual effective masses do not plateau until the $\sim 8^{\text {th }}$ timeslice, $M_{q}^{\lambda=2} / M_{q}^{\lambda=1}$ reaches the apparent plateau value of $\sim 90 \%$ by the $5^{\text {th }}$ timeslice and stays there.

propagators).

\section{MASSES AND GAUGE DEPENDENCE}

Since QED is infrared free, the electron mass $m_{e}$ appears as a pole of gauge invariant Greens functions consisting of an electron and positron separated widely enough that interaction between them is negligible. Then the pole of the electron propagator can be renormalized to be $m_{e}$. Since $m_{e}$ is derived from a gauge invariant Green's function, $m_{e}$ is manifestly gauge invariant. ${ }^{5}$

Due to photon fluctuations, the nonperturbative electron is a composite particle whose propagator has branch cuts rather than an isolated pole. To leading order in continuum perturbation theory, ${ }^{6}$ the Euclidean electron propagator is

$$
\int_{-\infty}^{+\infty} \frac{d p_{0}}{2 \pi} \frac{e^{i p_{0} t}}{\left(p_{0}^{2}+m_{e}^{2}\right)^{1+b}} \underset{\left(m_{e} t\right) \rightarrow \infty}{\rightarrow} A t^{-b} e^{-m_{e} t}
$$

where $b \equiv \alpha_{\mathrm{em}}(3-\xi) / 2 \pi$ and $\xi$ is the covariant gauge parameter. Consequently the effective mass $M_{e}=m_{e}+\frac{b}{t} \log t$ picks up a soft $\xi$ dependence. Nonetheless, the limiting value

$$
\lim _{t \rightarrow \infty} M_{e}=m_{e}
$$

is gauge invariant.

Since $Q C D$ is confining, it is not possible to isolate a manifestly gauge invariant $m_{q}$

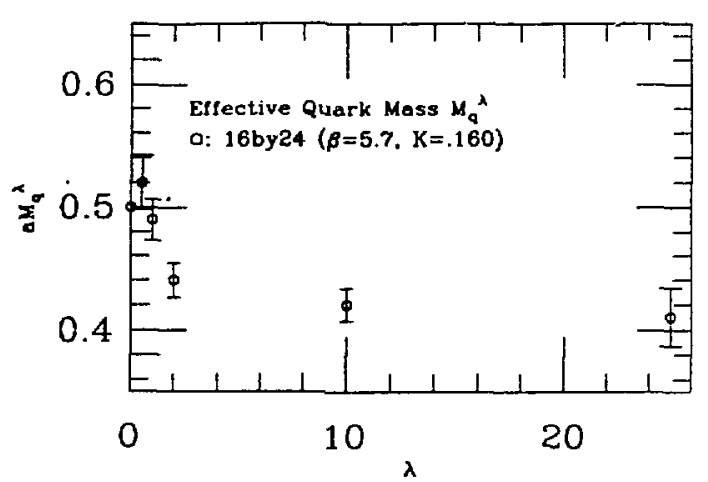

Figure 2: The effective quark mass decreases with increasing $\lambda$. Although not shown, this feature is reproduced on our $24 b y 40(\beta=6.0)$ lattices.

in gauge invariant Greens functions-constituent quark masses come with binding energy. What about extracting $m_{q}$ directly from the quark propagator? The problem is the QCD analogue of Eq. (8) is beyond perturbation theory, so it is not guaranteed that the $t \rightarrow \infty$ limit of $M_{q}$ is gauge invariant. Furthermore, since it is not measurable as the pole of gauge invariant Greens functions, $m_{q}$ may not even exist.

As a practical matter, even if Eq. (9) does hold for $Q C D$, the path to $m_{q}$ will be gauge dependent. Thus, unless the limit is reached quickly enough gauge invariance will not be observed within the lattice. Hence we study two questions: (i) How does $M_{q}$ depend on gauge? (ii)Does $M_{q}$ contain a gauge invariant component $m_{q}$ at large times?

Our $\lambda$ gauges are the lattice analogues of $\lambda \partial_{0} A_{0}+\nabla \cdot \vec{A}=0$ and are obtained by selecting gauge transformations $\Omega_{x}$ to drive

$$
\epsilon(\lambda, \mathcal{U}) \equiv \frac{1}{\# \text { sites }} \sum_{\mu, x} \operatorname{Tr}\left(\lambda_{\mu} \partial_{\mu} A_{\mu}\right)^{2}
$$

down by a factor of $10^{4}$ from the original configurations before gauge fixing ( $\Sigma$ gauge" 2 ). In (10), $\mathcal{U}$ represents the configuration of gauge links, and $\lambda_{0} \equiv \lambda, \lambda_{i} \equiv 1$. We have checked that the Landau gauge quark propagator is stable under an additional factor of 10 decrease in $\epsilon$.

$\lambda$ gaugs fixing can be viewed as freezing $\Omega_{x}$ fluctuations according to the $\xi \rightarrow 0$ limit of the 


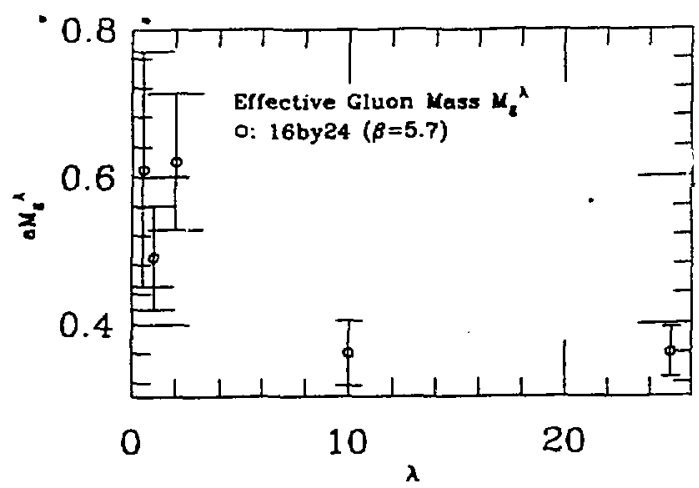

Figure 3: The effective gluon mass decreases with increasing $\lambda$. Although not shown, this feature is reproduced on our $24 b y 40(\beta=6.0)$ lattices.

action $^{7}$

$$
S_{\mathrm{gf}}=-\frac{1}{2 \xi} \sum_{x, \mu} \operatorname{Re} \operatorname{Tr}\left[\lambda_{\mu} \Omega_{x} U_{\dot{\mu}}(x) \Omega_{x+\dot{\mu}}^{\dagger}\right] .
$$

Then the $\lambda$ gauge quark propagator

$$
P(t)=\sum_{\bar{x}}<0\left|\Omega_{x} \psi(x) \bar{\psi}(0) \Omega_{0}^{\dagger}\right| 0>
$$

is an " $\Omega_{x} \psi(x)$ " propagator with respect to $\Sigma$ gauge. The gauge transformations $\Omega_{x}$-like quarks in the hopping constant $(K)$ expansionhop between neighboring sites with amplitude $\lambda_{\mu} / 2 \xi$ leaving behind a Wilson line trail. The integration over $\mathcal{U}$ suppresses all trails except those where the quark and $\Omega$ trails meet to form Wilson loops. In the strong coupling limit $(\beta \rightarrow 0$, $\xi \rightarrow \infty)$, only the timelike, arealess Wilson loops survive, and we get a mass $M_{q} \sim \log (N \xi / K \lambda)$, where $N$ is the number of colors (coming from $\Omega_{x}$ integrations). Therefore, at strong coupling $M_{q}$ decreases with increasing $\lambda$.

As Figures 1 and 2 show, our $\beta=5.7$ and $\beta=6.0$ numerical results follow this strong coupling trend. Though the dependence of $M_{q}$ on $\lambda$ is mild, it is statistically significant. Figure 3 shows that the effective gluon mass has similar behavior. Likewise $V_{h}$ (not shown) also decreases with increasing $\lambda .{ }^{4}$ Our Landau gauge gluon mass values $(\sim 500 \mathrm{MeV})$ are consistent with earlier lattice results ${ }^{1}$ and a continuum estimate. ${ }^{8}$

In an effort to uncover an underlying gauge invariant mass $m_{q}$, we have done correlated fits of

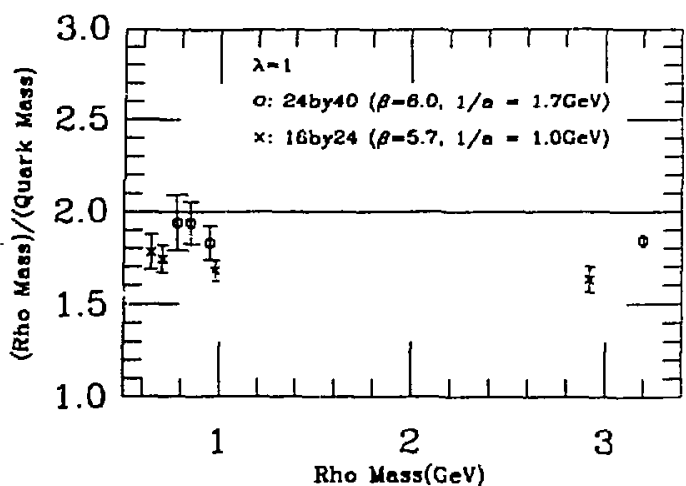

Figure 4: $M_{\rho} / M_{q}$ is independent of $K$ within our resolution. The $M_{p} / M_{q}=2$ line is to guide the eye.

the quark propagator to (i) $Z \mathrm{e}^{-M_{q} t}$, (ii) $A t^{B} \mathrm{e}^{-m_{\mathrm{q}} t}$ and (iii) $C \mathrm{e}^{-m_{\mathrm{q}} t}+D \mathrm{e}^{-m_{\mathrm{q}}^{\prime} t}$. In general, the propagator is not a good fit to (i) for the limited range of times available, but (ii) and (iii) with $D \sim-C$ fit well. ${ }^{4}$ Moreover, ratios of any two propagators (same K, different $\lambda$ ) fit well to $E t^{F}$. This suggests that $(i i)$ is a good fit for all available $\lambda$ when $m_{q}^{\lambda=\lambda_{0}}$ (for any choice of $\lambda_{0}$ ) is kept fixed and only $A$ and $B$ are varied with $\lambda$. However, when $A, B$ and $m_{\mathrm{q}}$ are simultaneously varied the best fit $m_{q}$ decreases with increasing $\lambda$.

Despite apparent gauge dependence, $M_{q}$ has some intriguing pheromenological features reminiscent of the nonrelativistic quark model. As noted previously, ${ }^{2} M_{q}^{\lambda=1} \sim M_{\text {proton }} / 3$. Additionally, the ratio of the $\rho$ mass $M_{\rho}$ to the quark mass $M_{q}$, depicted in Figure 4, is $\sim 2$ for all $K$. (Kilcup and Sharpe ${ }^{9}$ have found similar results.) The $1 \sigma$ to $2 \sigma$ scaling violation between $\beta=6.0$ and $\beta=5.7$ here is comparable to scaling violations seen in gauge invariant correlation functions. ${ }^{10}$ The reason for the difference from 2 of $M_{p} / M_{q}$ for large masses is unclear.

\section{3. $\Delta I=1 / 2$ RULE MATCHING}

Proportional to $\left(m_{u}-m_{c}\right) a^{-2}$, the $\bar{s} d$ coefficient in the lattice representation of the dimension-6 $\Delta S= \pm 1$ electroweak Hamiltonian is presumably not computable in weak coupling perturbation theory(WCPT) or any other conventional approach. ${ }^{11,12}$ We take advantage of 
gauge fixing to compute quark and gluon matrix elements from which such matching coefficients can be extracted.

Let $q$ be a quark of arbitrary flavor ( $q \in$ $\{u, d, s, c\})$ and define

$O_{ \pm}^{q q}\left[\Gamma_{1} \Gamma_{2}\right] \equiv \bar{s}(0) \Gamma_{1} d(0) \cdot \bar{q}(0) \Gamma_{2} q(0) \pm(d \leftrightarrow q)$.

The operators of interest are then $O_{ \pm}^{u u}\left[\Gamma_{1} \Gamma_{2}\right]-$ $O_{ \pm}^{\text {cce }}\left[\Gamma_{1} \Gamma_{2}\right]$. Their $K$ to $\pi$ matrix elements can be decomposed into "figure 8" and "eye" components.

In lowest order of chiral perturbation the ory(CPTh), the amplitude ratio for the $\Delta I=1 / 2$ part of $K_{s}^{0} \longrightarrow \pi^{+} \pi^{-}$divided by the $\Delta I=3 / 2$ part is given by 12

$$
\frac{3}{2} \times\left(\frac{1}{3}+\mathbf{R}_{2}^{+}+\frac{c_{-}}{c_{+}}\left(\mathbf{R}_{1}+\mathbf{R}_{2}^{-}\right)\right)
$$

where $c_{ \pm}$are $Q C D$ renormalization group coefficients,

$$
\begin{aligned}
& \mathbf{R}_{1} \equiv \frac{\left\langle O_{-}^{u u}[L L]\right\rangle_{\text {fig8 }}}{\left\langle O_{+}^{u u}[L L]\right\rangle_{\text {fig8 }}} \\
& \mathbf{R}_{2}^{ \pm} \equiv \frac{\left\langle O_{ \pm}^{u u}[L L]-O_{ \pm}^{\text {cc }}[L L]\right\rangle_{\text {eye }}}{\left\langle O_{+}^{u u}[L L]>_{\text {fig8 }}\right.}
\end{aligned}
$$

(with <> denoting a $K^{+} \mapsto \pi^{+}$matrix element), and the operators are renormalized to give zero $K \rightarrow 0$ transition amplitude. ${ }^{13}$

To compute the $\mathbf{R}_{1_{12}}^{ \pm}$on the lattice, it is necessary to know the coefficients $z_{ \pm}^{J}$ and $z_{ \pm}^{\Gamma_{1} \Gamma_{2}}$ in

$$
O_{ \pm}^{\text {cont }}[L L]=z_{ \pm}^{J} J^{\text {latt }}+\sum_{\Gamma_{1} \Gamma_{2}} z_{ \pm}^{\Gamma_{1} \Gamma_{2}} O_{ \pm}^{\text {latt }}\left[\Gamma_{1} \Gamma_{2}\right]
$$

where $J \equiv \bar{s}(0) d(0)$ and the sum over $\Gamma_{1} \Gamma_{2}$ is a sum over the combinations $L L, S S, P P, T T, L R$. Odd parity operators do not contribute and higher dimensional operators (order $a$ terms) are suppressed in the continuum limit and ignored here. The mixing with 4-quark operators in Eqn. (17) comes only from short distance effects in figure8 type graphs; mixing with penguin operators through eye graphs is absent ${ }^{14}$ due to GIM suppression. We neglect mixing with the color magnetic-moment operator $\bar{s} \sigma_{\mu \nu} F^{\mu \nu} d$ since it has a tiny perturbative coefficient ${ }^{15}$ (it comes in only in two loops) and is GIM suppressed.

We impose the matching condition

$$
<s\left|O_{ \pm}^{\text {cont }}[L L]\right| d>=0
$$

as the Wilson lattice equivalent, in the parity even sector, of the requirement that the $K \rightarrow 0$ amplitude vanish. We enforce the condition by individually subtracting $J^{\text {latt }}$ from each lattice operator $\left(\Theta^{\text {latt }} \rightarrow \Theta^{\text {latt }}-\alpha_{\Theta} J^{\text {latt }}\right)$ where the weight $\alpha_{\Theta}$ is defined by

$$
\left.\left.<s\left|\Theta^{\text {latt }}\right| d\right\rangle \equiv \alpha_{\Theta}<s\left|J^{\text {latt }}\right| d\right\rangle .
$$

Since $\mid s>$ and $\mid d>$ are not S-Matrix states, there is no guarantee that $\alpha_{\ominus}$ defined by Eq. (19) is related to $z_{ \pm}^{J}$ in Eq. (17). Note however that in an unconfined theory (18) would be precisely the correct condition since it prohibits the mixing of $s$ and $d$ quarks on shell. The best we can do here is to check that each $\alpha_{\ominus}$ has all the properties expected of $z_{ \pm}^{J}$ : (i)uniquenessthe scalar and vector components of the quark propagators in Eq. (19) give the same $\alpha_{\Theta}$; and (ii)gauge invariance- $\alpha_{\Theta}$ must be gauge invariant. We do not see any statistically significant violations of (i) or (ii). For example, at $\beta=6.0$ and $K=.154$

$$
\frac{\alpha_{O_{+}=2}^{\lambda=1}=1}{\alpha_{O_{+}[S S]}^{\lambda=1}}=1.00(.002) ; \frac{\alpha_{O_{-}[L L]}^{\lambda=2}}{\alpha_{O_{-}=1}^{\lambda=1}}=1.00(.092) .
$$

However, the real test of this method would be the scaling of the final continuum matrix elements after all subtractions have been made. Large fluctuations make that test meaningless at present.

Since $\bar{s} d$ doesn't contribute to figure 8 graphs, $<O_{ \pm}^{u u}[L L]>_{\text {figg }}$ isn't affected by subtraction. Following the technique ${ }^{16}$ used to correct $<\bar{K}^{0}\left|(\bar{s} d \bar{s} d)_{L L}^{\text {cont }}\right| K^{0}>$, the coefficients $z_{ \pm}^{\Gamma_{1} \Gamma_{2}} / z_{ \pm}^{L L}$ are determined by solving

$$
\sigma_{ \pm} p \cdot p=\sum_{\Gamma_{1} \Gamma_{2}} z_{ \pm}^{\Gamma_{1} \Gamma_{2}}<\left(O_{ \pm}^{u \mu}\left[\Gamma_{1} \Gamma_{2}\right]\right)^{\text {latt }}>_{\text {fig8 }}
$$

If one neglects mixing with the color magneticmoment eperator, $z_{ \pm}^{\Gamma_{1} \Gamma_{2}} / z_{ \pm}^{L L}$ for the eye graphs 


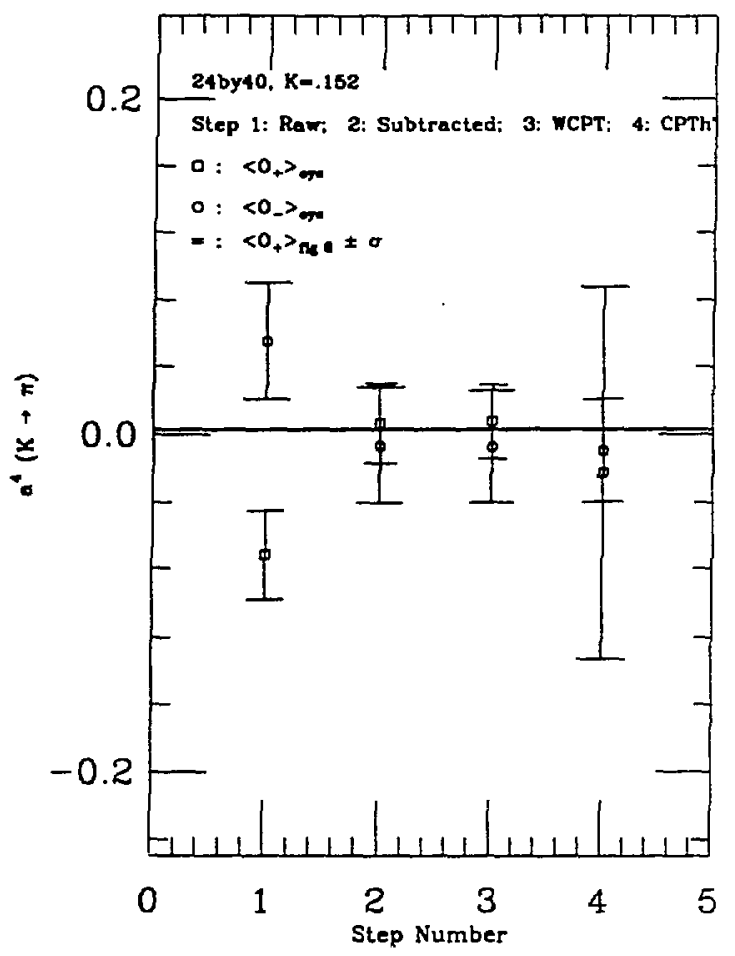

Figure 5: (eye graphs) $/ z_{ \pm}^{L L}$ at various stages. Step 1 is the raw lattice matrix elements $<O_{ \pm}^{\text {uu }}>_{\text {eye; }}$ step 2 is with $\bar{s} d$ subtraction only; step 3 is if WCPT values of $z_{ \pm}^{\Gamma_{1} \Gamma_{2}} / z_{ \pm}^{L L}$ are used to complete Eq. (17); step 4 is if CPTh behavior is enforced nonperturbatively using the figure8 graphs. The broad horizontal line is actually a pair of lines marking $\left(<O_{+}^{u u}>_{\text {fig8 }}+\sigma\right)$ and $\left(<O_{+}^{u u}\right\rangle_{\text {fig8 }}-\sigma$ ). Since the eye-graph errors are much larger than $\left\langle O_{+}^{u t}>_{\text {fig8 }} \pm \sigma\right.$, the $\mathbf{R}_{1,2}^{ \pm}$ values are washed out.

are the same as for the figure 8's. Alternatively, one can try just using the perturbative (weak coupling) coefficients. ${ }^{14}, 17$ Figure 5 depicts the answers from both techniques. Clearly much better statistics are needed before one can adequately test this approach.

\section{ACKNOWLEDGEMENTS}

We are grateful to Jeff Mandula, Michael Ogilvie, Greg Kilcup, Steve Sharpe and Mike Cornwall for discussions. The computing was done at the National Energy Research Supercomputer Center (partially within "Grand Challenge") and the San Diego Supercomputer Center.

\section{REFERENCES}

1. J. Mandula and M. Ogilvie, Phys. Lett. B185 (1987) 127; see also: R. Gupta, G. Guralnik, G. Kilcup, A. Patel, S. Sharpe and T. Warnock, Phys. Rev. D36, 2813 (1987).

2. C. Bernard, D. Murphy, A. Soni, K. Yee, Nucl. Phys. B(Proc. Suppl.) 17 (1990) 593

3. For studies in $U(1)$ see $P$. Coddington, $A$. Hey, J. Mandula and M. Ogilvie, Phys. Lett. B197 (1987) 191; A. Nakamura and R. Sinclair, Phys. Lett. B243 (1990) 396; B. Berg and Panogiotakopoulos, Phys. Rev. Lett. 52 (1984) 94

4. C. Bernard, A. Soni, K. Yee, in progress.

5. S. Coleman, Lecture \#8 (particularly pp. 385-386 of 1988 paperback edition) in Aspects of Symmetry, Cambridge University Press, Cambridge, GB (1985)

6. H. M. Fried, Functional Methods and Models in Quantum Field Theory, MIT Press, Cambridge, MA (1972)

7. M. Ogilvie, Phys. Lett. B231 (1989) 161, and private communications.

8. J. M. Cornwall, Phys. Rev. D26 (1982) 1453.

9. G. Kilcup and S. Sharpe, private communication

10. See, for example, C. Bernard, A. El-Khadra and $A$. Soni, preprint BNL-45157, NSF-ITP. 90-147 (to be submitted to Phys. Rev. D).

11. L. Maiani, G. Martinelli, G. C. Rossi and M. Testa, Phys. Lett. 176B (1986) 445

12. C. Bernard in T. Degrand and D. Toussaint, eds., From Actions To Answers World Scientific, 1990 and references therein.

13. C. Bernard, T. Draper, H. Politzer, A. Soni, and M. Wise, Phys. Rev. D32 (1985) 2343.

14. C. Bernard, T. Draper, A. Soni, Phys. Rev. D36 (1987) 3224.

15. G. Curci, et al., Phys. Lett. 202B (1988) 363.

16. C. Bernard and A. Soni, Nucl. Phys. B(Proc. Suppl.) 17 (1990) 495.

17. G. Martinelli, Phys. Lett. 141B (1984) 395. 